\title{
ODONTOMA COMPOSTO EM ADULTO E SUA COMPLEXIDADE DE TRATAMENTO: RELATO DE CASO CLÍNICO
}

\author{
COMPOUND ODONTOMA IN ADULT AND ITS TREATMENT COMPLEXITY: CLINICAL CASE REPORT
}

\section{Sebastião Silvério de SOUSA-NETO'; Gimenna Barbosa PEREIRA²; Weberson Dantas LEMES²; Vitor Henrique Carvalho SILVA3; Cláudio Souto França FELGA3; Allisson Filipe Lopes MARTINS ${ }^{4}$}

1 - Acadêmico do curso de Odontologia da Faculdade de Odontologia da Universidade Federal de Goiás, Goiânia/GO - Brasil.

2 - Acadêmico do curso de Odontologia da Faculdade União de Goyazes, Trindade/GO - Brasil.

3- Cirurgião Bucomaxilofacial, Goiânia/GO - Brasil.

4 - Pesquisador Institucional e Professor de Diagnóstico Bucal da Faculdade União de Goyazes, Trindade/GO - Brasil.

\section{RESUMO}

Introdução: Odontoma é o tumor odontogênico mais frequente e é diagnosticado durante as primeiras décadas de vida, devido ao atraso na erupção de um dente ou por meio de exame radiográfico de rotina. As características radiográficas e microscópicas são úteis para sua classificação em complexo ou composto. Odontomas em pacientes adultos são incomuns e podem ser complexos de tratar. Objetivo: O objetivo deste trabalho foi relatar a complexidade de tratamento de um odontoma composto em paciente adulto. Material e Método: o presente artigo é do tipo relato de caso. Resultados: Paciente do sexo feminino, 32 anos, compareceu ao cirurgião-dentista para avaliação clínica de rotina. Durante o exame clínico foi observada a ausência do dente 43 , exames radiográficos demonstraram lesão mista, bem delimitada, com estruturas semelhantes a dentículos, localizada na região anterior de mandíbula do lado direito, entre as raízes dos dentes 42 e 44, promovendo deslocamento do dente 43 para a base da mandíbula. Devido à localização da lesão e seus efeitos no dente 43 , a paciente foi submetida à ressecção da lesão sob anestesia geral. Foi realizada osteotomia na tábua óssea vestibular da mandíbula, remoção de múltiplas estruturas semelhantes a dentes, instalação de placa de reconstrução mandibular sistema 2.4 com cinco parafusos do mesmo sistema e enxerto ósseo com biomaterial aloplástico. O dente 13 foi extraído. As radiografias de controle pós-operatório evidenciaram bom posicionamento do material. O controle pós-operatório indica que a paciente encontra-se bem e sem ausência de sinais de recidiva ou complicações cirúrgicas. Conclusão: O diagnóstico tardio de odontomas associado ao deslocamento dentário ocasionado pelo tumor podem tornar o seu tratamento mais complexo.

Palavras-chave: Tumores Odontogênicos; Odontoma; Reconstrução Mandibular.

\section{INTRODUÇÃO}

A primeira classificação de tumores odontogênicos foi realizada em 1868 pelo médico francês Pierre Paul Broca, que utilizou o termo odontoma para se referir a todos os crescimentos tumorais desenvolvidos a partir dos tecidos formadores do aparato dentário ${ }^{1}$. Os odontomas, de acordo com a atual classificação da Organização Mundial de Saúde (OMS), são tumores odontogênicos de origem mista, ou seja, possuem componentes epiteliais e mesenquimais odontogênico ${ }^{2}$. Esses tumores podem ser subclassificados em complexos, quando formam massas disformes de material semelhante a esmalte e dentina, ou compostos quando formam dentículos múltiplos com presença inclusive de tecido semelhante à polpa dentária² .

Em geral, odontomas são lesões assintomáticos, que apresentam sutil predileção pela região anterior de maxila em relação à região anterior da mandíbula ${ }^{3-6}$. Frequentemente, ocasionam impacção e/ou mau posicionamento dentário, além de possível aumento de volume no local afetado. Devido à ausência de sintomas são ocasionalmente diagnosticados na investigação causal da ausência dentária ou em exames radiográficos de rotina ${ }^{7,8}$.
O odontoma destaca-se como o tumor odontogênico mais frequente em populações americanas, inclusive em populações brasileiras, como relatado por Santos et al. ${ }^{9}$ (2001) e Servato et al. $^{10}$ (2012). Em populações africanas, entretanto, os ameloblastomas correspondem aos tumores mais frequentes ${ }^{11,12}$. Essa marcante discrepância foi associada ao subdiagnóstico e ao baixo número de casos relatados ${ }^{11-13}$.

O tratamento de escolha para o odontoma é a enucleação cirúrgica, tal como descrito por Gold et al. ${ }^{14}$ (1991), seguida de envio do espécime ao exame anatomopatológico ${ }^{14}$. $\mathrm{O}$ tratamento cirúrgico pode ser agressivo, principalmente em lesões extensas e diagnosticadas tardiamente em mandíbula, elevando o risco de fratura mandibular ${ }^{15-17}$. A indicação terapêutica nesses casos envolve a utilização de placas de reconstrução e enxerto ósseo, a fim de melhorar o prognóstico do tratamento, no entanto, são considerados procedimentos cirúrgicos delicados e de maior complexidade ${ }^{15-17}$. Portanto, o objetivo do presente trabalho é relatar um caso de tratamento cirúrgico desafiador de odontoma composto em mandíbula de uma paciente adulta. 


\section{RELATO DO CASO}

Paciente do sexo feminino, 32 anos, feoderma, compareceu à clínica odontológica para consulta de rotina. A história médica pregressa não revelou histórico de traumas ou doenças de base. Durante o exame clínico intraoral foi observada a ausência do dente 43. Foi solicitada radiografia panorâmica que demonstrou dente canino deslocado para a base mandibular, associado à lesão radiopaca com halo radiolúcido, sugerindo odontoma composto.

Para melhor estudo e planejamento cirúrgico do caso, foi solicitada uma tomografia computadorizada de feixe cônico. Os cortes e reconstruções tomográficas demonstraram lesão mista (hiper- e hipodensa), medindo aproximadamente $15 \mathrm{~mm}$ em seu maior diâmetro, bem delimitada, formando estruturas semelhantes a dentes, localizada em corpo mandibular do lado direito, entre os dentes 42 e 44 , com deslocamento do dente 43 para a base da mandíbula, ocasionando adelgaçamento da cortical da base da mandíbula (Figura 1A a J). A partir disso foi indicado à realização de enucleação da lesão e extração do dente 43 e, devido ao risco de fratura mandibular em decorrência da exodontia, instalação de placas de reconstrução mandibular e enxertia do leito cirúrgico.

A paciente foi tratada em centro cirúrgico sob anestesia geral e intubação nasotraqueal. Foi realizada antibioticoprofilaxia no momento da indução pelo médico anestesista, aplicando, via endovenosa, $2 \mathrm{~g}$ de cefazolina. Após indução da paciente, foi realizada infiltração anestésica com vasoconstritor (cloridrato de lidocaína a 2\% com epinefrina 1:100.000) em região de fundo de vestíbulo mandibular seguida de incisão no local. Após divulsão dos tecidos e descolamento subperiosteal, o nervo mentual foi identificado e preservado (Figura 2). Foi realizada a osteotomia em base de mandíbula com broca cirúrgica $n^{\mathrm{o}} 702$ com intuito de promover exposição e possibilitar a remoção do dente 43 . Seguiu-se com osteotomia na região da lesão e remoção de múltiplas estruturas semelhantes a dentes (Figura 3). Para a síntese óssea foram instaladas placas de reconstrução mandibular sistema 2.4 com cinco parafusos do mesmo sistema (Jeil Medical Corporation, Coreia do Sul), além de enxertia do sítio cirúrgico com biomaterial aloplástico granulado Geistlich Bio-Oss ${ }^{\circledR}$ (Geistlich Pharma do Brasil, São Paulo), cobertura com membrana Geistlich Bio-Gide ${ }^{\circledR}$ (Geistlich Pharma do Brasil, São Paulo) e sutura contínua com fio absorvível monocryl. Como medicação pós-operatória, foi prescrito amoxicilina $500 \mathrm{mg}$ de oito em oito horas por sete dias, ibuprofeno $600 \mathrm{mg}$ de oito em oito horas por três dias e dipirona sódica $500 \mathrm{mg}$ de seis em seis horas por três dias, em caso de dor.

O material enucleado foi enviado para exame anatomopatológico que confirmou a hipótese diagnóstica de odontoma composto. A paciente retornou após sete dias com bom aspecto cicatricial, ausência de sinais flogísticos e relatando leve, e já esperada, parestesia do nervo mentual. Após um mês, paciente relatou regressão total da parestesia. As radiografias extraorais de controle pós-operatório evidenciaram bom aspecto da região operada (Figura 4). A paciente está sobre controle há 4 meses, sem sinais de recidiva.

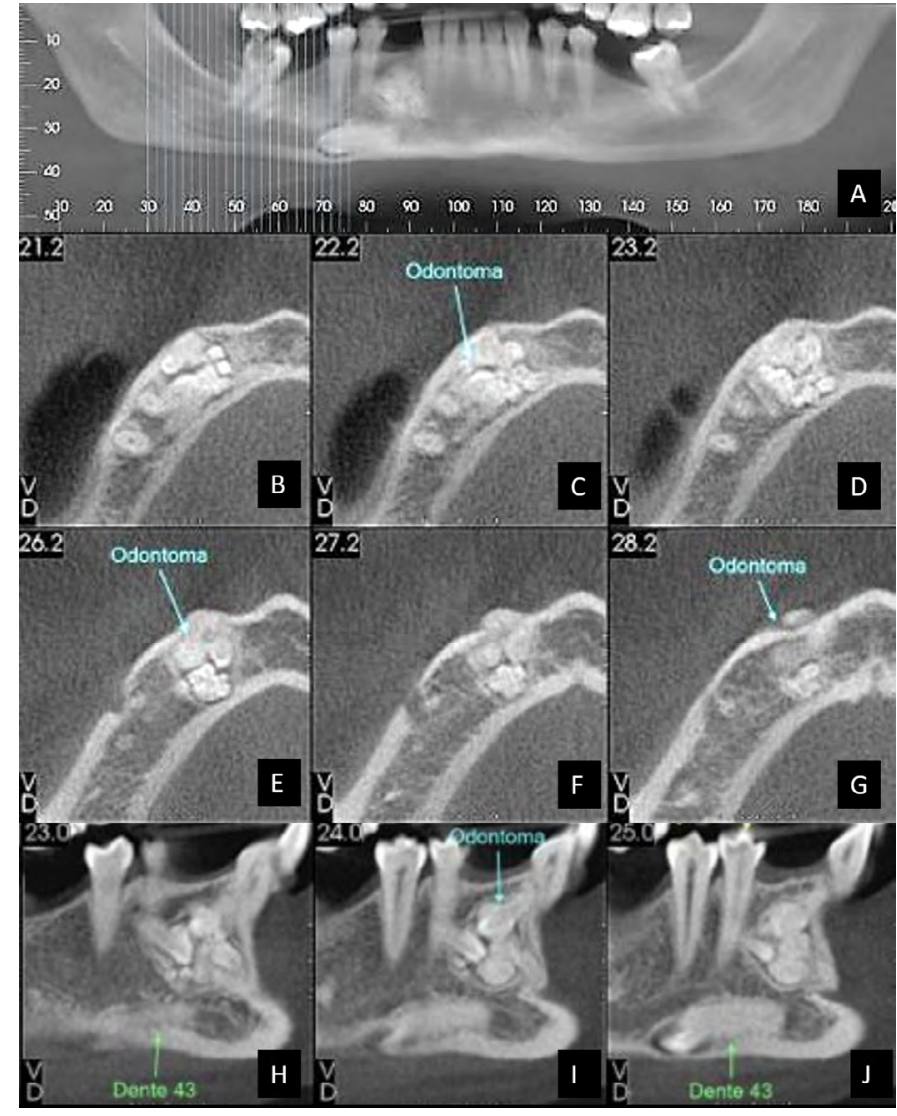

Figura 1 - Tomografia Computadorizada por Feixe Cônico da região. Em (A) Reconstrução panorâmica evidenciando posição ântero-posterior do odontoma e do dente 43 impactado. Em (B), (C), (D), (E), (F) e (G) cortes axiais demonstrando lesão mista, bem delimitada, com estruturas semelhantes à dentículos. Evidencia-se também o comprometimento em toda extensão vestíbulo-lingual nas zonas neutra e de tensão da região do dente 43. Em (H), (I) e (J) cortes transversais demonstrando lesão mista, medindo aproximadamente $15 \mathrm{~mm}$ em seu maior diâmetro, bem delimitada, corticalizada, localizada em corpo mandibular do lado direito, entre os dentes 42 e 44, com deslocamento do dente 43 para a base da mandíbula, ocasionando adelgaçamento da cortical, evidencia-se novamente estruturas semelhantes à dentes.

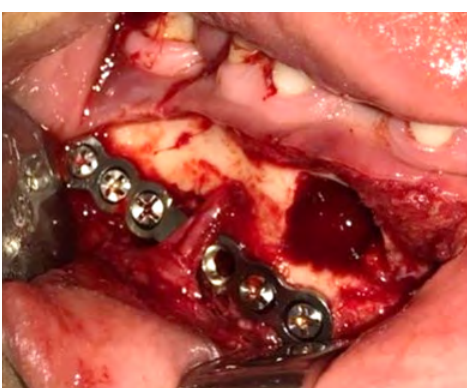

Figura 2 - Nervo mentual direito em destaque, preservado após enucleação da lesão e instalação de placa de reconstrução mandibular sistema 2.4 com cinco parafusos do mesmo sistema (Jeil Medical Corporation, Coreia do Sul).

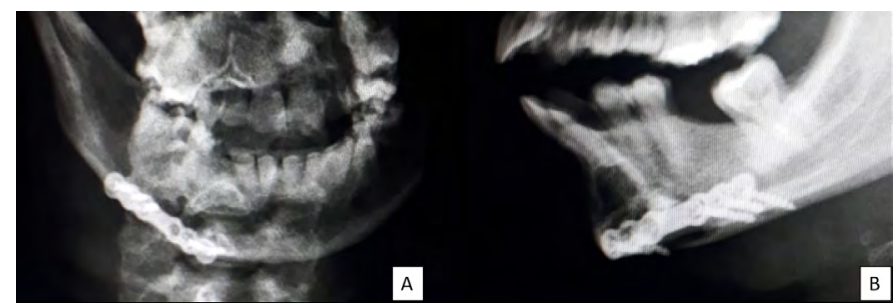

Figura 4 - Radiografias PA de face (A) e lateral de mandíbula (B), evidenciando bom aspecto da região operada.

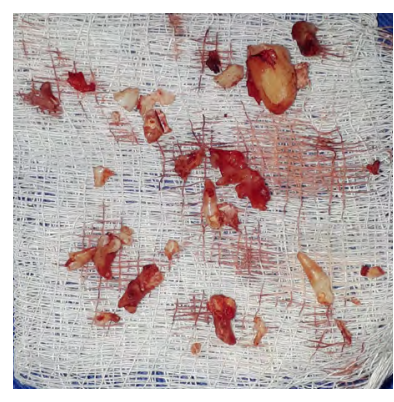

Figura 3 - Múltiplos estruturas semelhantes à dentes e dente 43 removidos. 


\section{DISCUSSÃO}

O odontoma é um tumor odontogênico benigno que acomete principalmente as duas primeiras década de vida, sem predileção por sexo². Um dos sinais clínicos associados a essa lesão é o atraso na erupção dentária. No presente caso uma paciente de 32 anos apresentava ausência do dente 43 , que nos radiográficos apresentava-se deslocado em posição horizontal na base da mandíbula do lado direito, sugerindo um tempo de desenvolvimento tumoral longo e provável negligência de outros profissionais quanto à orientação da paciente, pois, de acordo com Feraru et al. ${ }^{18}$ (2011), o período médio de erupção dos caninos inferiores em meninas é por volta dos 10 anos de idade ${ }^{18}$. Apesar de classificados como tumores, tem-se levantado a hipótese de que essas lesões são na verdade hamartomas ou lesões do desenvolvimento, não representando verdadeiras neoplasias ${ }^{19,20}$.

Blinder et al. ${ }^{21}$ (1993) descreveu as possíveis técnicas cirúrgicas para tratamento de odontomas em ângulo mandibular e dentre elas a excisão intraoral e acesso à lesão via cortical bucal. Apesar de o acesso intraoral evitar a formação de cicatrizes e facilitar o ato cirúrgico, essa técnica apresenta risco de fratura da delgada cortical lingual após a enucleação da lesão ${ }^{21}$.

Kuramochi et al. ${ }^{15}$ (2006) descreveu a utilização da técnica de osteotomia vestibular a partir de incisão extraoral para remoção de odontoma complexo em corpo-ângulo mandibular esquerdo, no entanto, essa técnica promove cicatrizes faciais, que podem ser vistas como defeitos estéticos ${ }^{15}$. No presente caso a conduta cirúrgica foi a de acesso intraoral e osteotomia por vestibular, corroborando com nosso trabalho, Nogueira et al. ${ }^{17}$ (2013) também utilizou a corticotomia vestibular para tratamento de odontoma em corpo e ramo mandibular, descrevendo a técnica como alternativa terapêutica ao acesso extraoral, apresentando excelentes resultados pós-operatórios ${ }^{17}$.

Finalmente, o presente relato demonstrou que a paciente apresentava dente canino deslocado para a base mandibular, ocasionando adelgaçamento dessa cortical basal da mandíbula, que poderia levar à fratura devido à enucleação da lesão e remoção do dente, portanto foi indicado também o sistema de fixação óssea com placas e parafusos. Semelhante ao caso apresentado, Kuramochi et al. ${ }^{15}$ (2006) descreveu a conduta e tratamento de um odontoma complexo extenso de corpo-ângulo mandibular no qual ocorreu fratura da cortical basal, sendo necessário o reposicionamento e estabilização com placa sistema $2.4^{15}$. A AO Foundation, uma organização internacional de cirurgiões que atuam no tratamento de traumas e distúrbios musculoesqueléticos, indica a utilização de placas de reconstrução do sistema 2.4 na zona neutra, seguindo as linhas ideais de osteossíntese preconizadas por Champy, em casos de fratura simples em corpo mandibular ${ }^{23}$. Neste caso optou-se pelo sistema load bering, com utilização de placa sistema 2.4, devido ao comprometimento da base da mandíbula.

Apesar de ser uma lesão benigna indolente, se não tratado, o odontoma pode permanecer intraósseo por vários anos, levando ao desenvolvimento de lesões extensas que requerem tratamentos cirúrgicos agressivos, complexos e deformantes ${ }^{15,16,21,22}$. O cirurgião-dentista deve estar atento às ausências dentárias, devendo solicitar exames complementares para investigação dessa ausência, o que possibilita o diagnóstico precoce e tratamento cirúrgico conservador de alterações intraósseas relacionadas à impacção dentária.

\section{CONCLUSÃO}

O presente caso ilustra que o diagnóstico e tratamentos tardios de odontomas podem resultar em cirurgias mais complexas que requerem uso de elementos de fixação óssea e enxertos, encarecendo o tratamento e exigindo técnica cirúrgica mais apurada.

\section{REFERÊNCIAS}

01. Henriksson CO, Kjellman O. Complex odontoma: report of a case. Oral Surg Oral Med Oral Pathol. 1964; 18(1):64-69.

02. World Health Organization. Classification of tumours. Lyon: IARC Publishing Group; 2017.

03. Tekkesin MS, Pehlivan S, Olgac V, et al. Clinical and histopathological investigation of odontomas: review of the literature and presentation of 160 cases. J Oral Maxillofac Surg. 2012; 70(6):1358-1361.

04. Ochsenius G, Ortega A, Godoy L, Penafiel C, Escobar E. Odontogenic tumors in Chili: a study of 362 cases. J Oral Pathol Med. 2002; 31(7): 415-20.

05. Mosqueda-Taylor A, Ledesma-Montes C, Caballero-Sandoval S, Portilla-Robertson J, Ruiz-Godoy RLM, Meneses-Garcia A. Odontogenic tumors in Mexico: a collaborative retrospective study of 349 cases. Oral Surg Oral Med Oral Pathol Oral Radiol Endod. 1997; 84(6): 672-5.

06. Philipsen H, Reichart P, Praetorius F. Mixed odontogenic tumours and odontomas. Considerations on interrelationship. Review of the literature and presentation of 134 new cases of odontomas. Oral Oncol. 1997; 33(2): 86-99.

07. López-Areal L, Silvestre-Donat F, Gil-Lozano J. Compound odontoma erupting in the mouth: 4 - year follow-up of a clinical case. J Oral Pathol Med. 1992; 21(6): 285-8.

08. Fregnani ER, Fillipi RZ, Oliveira CRGCM, Vargas PA, Almeida OP. Odontomas and ameloblastomas: variable prevalences around the world? Oral Oncol. 2002; 38(8): 807-808

09. Santos JN, Pinto LP, Figueredo CR, Souza LB. Odontogenic tumors: analysis of 127 cases. Pesqui Odontol Bras. 2001; 15(4): 308-13.

10. Servato JPS, Souza PEA, Horta MCR, Ribeiro DC, Aguiar MCF, Faria PR, et al. Odontogenic tumours in children and adolescents: a collaborative study of 431 cases. Int J Oral Maxillo Fac Surg. 2012; 41(6): 768-773.

11. Adebayo ET, Ajike SO, Adeyeke EO. Tumors and tumor like lesions of the oral and perioral structures of Nigerian children. Int J Oral MaxillofacSurg. 2001; 30(3): 205-8.

12. Ladeinde AL, Ajayi OF, Ogunlewe MO, et al. Odontogenic tumors: a review of 319 cases in a Nigerian teaching hospital. Oral Surg Oral Med Oral Pathol Oral Radiol Endod. 2005; 99(2): 191-5.

13. Aregbsola B, Soyele O, Effiom O, Gbotolorun O, Taiwo O, Amole I. Odontogenic tumours in Nigeria: A multicenter study of 582 cases and review of literature. Med Oral Patol Oral Cir Bucal. 2018; 23(6): e761-e766.

14. Gold L, Upton GW, Marx RE. Standardized surgical terminology for the excision o lesions in bone. An argument for accuracy in reporting. J Oral Maxillofac Surg. 1991; 49(11):1214.

15. Kuramochi MM, Vanti LA, Berenguel IA, Pereira WL, Zangrando D. Acesso extraoral para reconstrução primária em odontoma complexo raro na mandíbula. Rev Port Estomat Med Dent Cir Maxilof. 2006; 47(1): 35-40.

16. Casap N, Zeltser R, Abu-Tair J, Shteyer A. Removal of a large odontoma by sagittal split osteotomy. J Oral Maxillofac Surg. 2006; 64(12): 1833. 
17. Nogueira AS, Gonçales ES, Gonçales AG, Thiegui-Neto V, Nogueira CB, Nogueira A, Medeiros R. Surgical treatment of extensive complex odontoma in the mandible by corticotomy. A case report. Minerva Stomatol. 2013; 62(9): 335-42.

18. Feraru IV, Rãducanu AM, Feraru SE, Herpeliu C. The sequence and chronology of the eruption of permanent canines and premolars in a group of Romanian children in Bucharest. Oral Health and Dental Management. 2011; 10(4): 193-198.

19. Veis A, Tziafas D, Lambrianidis T. A case report of a compound odontoma causing delayed eruption of a central maxillary incisor: clinical and microscopic evaluation. J Endod. 2000; 26(8): 477-9.
20. Madeira AA, Minatti EJ. Odontomas complexo e combinado. Odontol Mod. 1985; 12(5): 32-8.

21. Blinder D, Peleg M, Taicher S. Surgical considerations in cases of large mandibular odontomas located in mandibular angle. Int J Oral Maxillofac Surg. 1993; 22(3): 163-5.

22. Amado CS, Gargallo AJ, Berini AL, Gay C. Revisión de 61 casos de odontoma. Presentación de un odontoma complejo erupcionado. Med Oral. 2003; 8: 366-73.

23. Colton C, Orson J. (2013). Plates: form and function. [online]. [Acesso em 26 de setembro de 2019]. Disponível em: https://aotrauma. aofoundation.org//media/project/aocmf/aotrauma/documents/ education_pdf.

\section{ABSTRACT}

Introduction: The most common odontogenic tumor is Odontoma, and is diagnosed during the first decades of life, due to a delayed eruption of a tooth, or through routine radiographic examination. From the radiographic and microscopic characteristics is classified into complex or compound. Odontomas in adult patients are uncommon and can have a complex treatment. Aim: The aim of this study was to report the complexity of treating a compound odontoma in an adult patient. Material and Methods: the present research is a case report. Results: A 32-year-old female patient attended the dental surgeon for routine evaluation. During the clinical examination, the absence of tooth 43 was observed, image exams revealed a well-defined mixed lesion consisting of denticles, located in the anterior region of the right mandible, between the roots of the teeth 42 and 44, the lesion promoted displacement of tooth 43. Due to the location and its effects on tooth 43 , the patient underwent resection of the lesion under general anesthesia. Surgery proceeded with buccal plate osteotomy, removal of multiple tooth-like structures, installation of mandibular reconstruction plates 2.4 with five screws and bone graft with alloplastic biomaterial. Tooth 13 was extracted. Postoperative control radiographs showed good positioning of the material. Postoperative control show that patient does not have recurrence signal or complications due to surgery. Conclusion: Late diagnosis of odontomas associated with tooth displacement caused by the tumor may make its treatment more complex.

Keywords: Odontogenic Tumors; Odontoma; Mandibular Reconstruction.

\section{AUTOR PARA CORRESPONDÊNCIA}

Allisson Filipe Lopes Martins

Faculdade União de Goyazes, Trindade, Goiás, Brasil.

E-mail: allissonfilipe@hotmail.com

Telefone: +55 62981245239 